\title{
CHEMICAL CONSTITUENTS FROM LEAVES OF SONNERATIA ALBA \\ J.E. SMITH (SONNERATIACEAE)
}

\author{
Nguyen Thi Hoai Thu ${ }^{(1)}$, Lam Phuc Khanh ${ }^{(1)}$, Nguyen The Duy ${ }^{(1)}$, \\ Nguyen Thi Kim Chanh ${ }^{(1)}$, Nguyen Kim Phi Phung ${ }^{(1)}$, Poul Erik Hansen ${ }^{(2)}$
}

(1) University of Science, VNU-HCM

(2) Roskilde University

(Manuscript Received on January $24^{\text {th }}$ 2011, Manuscript Revised March $28^{\text {th }} 2011$ )

\begin{abstract}
Sonneratia alba J.E. Smith, Sonneratiaceae widely grows in mangrove forests. There were some studies on plants of mangrove forests, and these results showed they contained many interesting bioactive compounds. Nevertheless, Sonneratia alba has not much been studied, especially, has not yet been chemically and biologically studied in Viet Nam. From the petroleum ether extract of the leaves of Sonnertia alba, oleanolic acid (1), betulin (2), betulinic acid (3), alphitolic acid (4), methyl gallate (5) and 5-hydroxymethylfurfural (6) were isolated. Their structures were identified by comparing their NMR data as well as the physical properties with those in literatures. Among them, (1) had high yield (about $0.15 \%$ of dried leave) with numerous pharmacological activities including inhibitory activity against $H I V-1$ protease with $I_{50}$ of $6.3 \mu \mathrm{M}$, anti-inflammatory, anti-cancer. So, Sonneratia alba should be exploited to afford to a valuable source in food and pharmaceutical products. Further studies on this plant are in progress.
\end{abstract}

Key words: Sonneratiaceae, Sonneratia alba, oleanolic acid, betulin, betulinic acid, alphitolic acid, methyl gallate, 5-hydroxymethylfurfural.

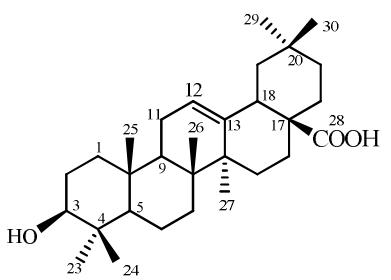

(1)

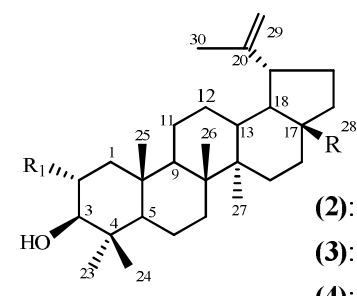

(2): $\mathrm{R}=\mathrm{CH}_{2} \mathrm{OH}$

(3): $\mathrm{R}=\mathrm{COOH}$;

(4): $\mathrm{R}=\mathrm{COOH}$;
$\mathrm{R}_{1}=\mathrm{H}$

$\mathrm{R}_{1}=\mathrm{H}$

$\mathrm{R}_{1}=\mathrm{OH}$<smiles>CC(=O)c1cc(O)c(O)c(O)c1</smiles>

(5)
(6)

\section{INTRODUCTION}

Sonneratia alba J. E. Smith, Sonneratiaceae (SA, Fig. 1) wildly grows in many mangrove forests in Viet Nam. There were some studies on plants of mangrove forests, and these results showed plants of mangrove forests contained many interesting bioactive compounds such as triterpeneoids, steroids, glycosides, flavonoids, alkaloids, quinonoids... Nevertheless, SA has not much been studied, especially, not yet in Viet Nam. In this paper, the isolation and structural determination of six compounds: oleanolic acid (1), betulin (2), betulinic acid (3), alphitolic 
acid (4), methyl gallate (5) and 5hydroxymethylfurfural (6) were reported. Among them, (1) was already known in fruits of Sonneratia ovata Back. and (2), (5) were already isolated from stems and twigs of Sonneratia caseolaris (L.) Engl.

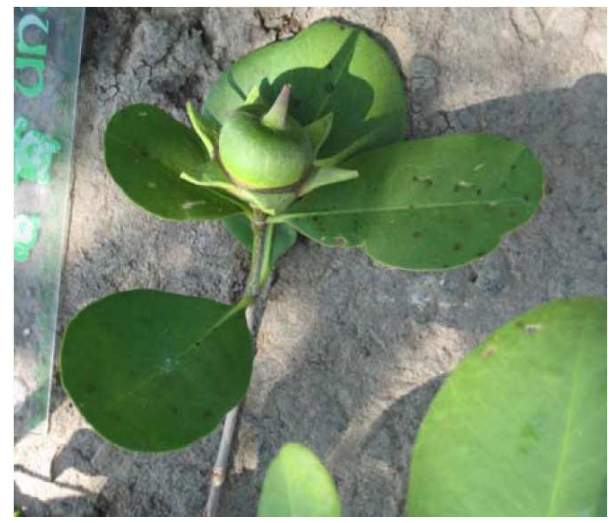

Figure 1. Sonneratia alba J.E. Smith

\section{MATERIALS AND METHODS}

\section{Plant materials}

Fresh leaves of the plant were collected in Can Gio mangrove forest in Ho Chi Minh City, Viet Nam in December 2009. The scientific name of the plant was identified by Phan Duc Binh, pharmacist, Associate Editor-in-Chief of the Journal of Drugs and Health. A voucher specimen (No US-B005) was deposited in the herbarium of the Department of Organic Chemistry, University of Science, Vietnam National University - Ho Chi Minh City.

\section{Extraction and isolation}

Fresh leaves $(28.0 \mathrm{~kg})$ were washed, dried, ground into powder $(13 \mathrm{~kg})$ and were extracted by percolation with methanol at room temperature then the methanol extract was evaporated in vacuo to give a methanol residue
$(2 \mathrm{~kg})$. This crude was suspended in water and partitioned against petroleum ether to afford petroleum ether residue $(\mathbf{E}, 700 \mathrm{~g})$. The residue E was chromatographed (on a column of si-gel) to afford six compounds (1), (2), (3), (4), (5) and (6).

\section{Oleanolic acid (1)}

Colourless amorphous powder, mp. $310{ }^{\circ} \mathrm{C}$ $\left(\mathrm{CHCl}_{3}\right)$. The ${ }^{1} \mathrm{H}$ - and ${ }^{13} \mathrm{C}$-NMR: See Table 1.

\section{Betulin (2)}

Colourless amorphous powder, mp. 256$257{ }^{\circ} \mathrm{C}\left(\mathrm{CHCl}_{3}\right)$. The ${ }^{1} \mathrm{H}$ - and ${ }^{13} \mathrm{C}-\mathrm{NMR}$ : See Table 1.

\section{Betulinic acid (3)}

Colourless amorphous powder, mp. 316$318{ }^{\circ} \mathrm{C}\left(\mathrm{CH}_{3} \mathrm{OH}\right)$. The ${ }^{1} \mathrm{H}$ - and ${ }^{13} \mathrm{C}-\mathrm{NMR}$ : See Table 1.

\section{Alphitolic acid (4)}

Colourless amorphous powder, mp. 310$314{ }^{\circ} \mathrm{C}\left(\mathrm{CH}_{3} \mathrm{OH}\right)$. The ${ }^{1} \mathrm{H}$ - and ${ }^{13} \mathrm{C}-\mathrm{NMR}$ : See Table 1.

\section{Methyl gallate (5)}

Colourless amorphous powder, mp. 201$203{ }^{\circ} \mathrm{C}\left(\mathrm{CH}_{3} \mathrm{OH}\right)$. The ${ }^{1} \mathrm{H}-\mathrm{NMR}, \mathrm{CD}_{3} \mathrm{OD}$, Sppm: 7.01 (2H, s, H-2, H-6), $3.82(3 \mathrm{H}, \mathrm{s}$, $\mathrm{CH}_{3}$ ). The ${ }^{13} \mathrm{C}-\mathrm{NMR}, \mathrm{CD}_{3} \mathrm{OD}, \delta$ ppm: 169.1 (COO-), 146.5 (C-3, C-5), 139.8 (C-4), 121.6 (C-1), 110.1 (C-2, C-6), 52.3 ( (HH $\left._{3}-\mathrm{O}-\right)$.

\section{5-Hydroxymethylfurfural (6)}

Colourless wax. The ${ }^{1} \mathrm{H}-\mathrm{NMR}, \mathrm{CDCl}_{3}$, Sppm: 6.59 (1H, d, 3.0, H-3), 7.39 (1H, d, 3.0, $\mathrm{H}-4), 9.60$ (1H, s, -Cㅌㅇ), $4.91(2 \mathrm{H}$, s, $\left.\mathrm{CH}_{2} \mathrm{OH}\right)$. The ${ }^{13} \mathrm{C}-\mathrm{NMR}, \mathrm{CDCl}_{3}$, $\delta$ ppm: 153.4 
(C-2), 123.7 (C-3), 110.2 (C-4), 163.0 (C-5), 178.2 (-ㅡH), $57.5\left(-\underline{C H}_{2} \mathrm{OH}\right)$.

\section{RESULTS AND DISCUSSION}

Compound (1) was a triterpenee with 30 carbons in the ${ }^{13} \mathrm{C}$-NMR spectrum. Based on two olefinic carbon signals at $\delta 143.6$ and 122.6 and a singlet olefinic proton signal at $\delta$ 5.27 as well as seven singlet methyl proton signals at high resonance field showed that (1) was a triterpene with olean-12-en skeleton. Resonance at $\delta 79.0(\mathrm{O}-\underline{\mathrm{CH}})$ was oxygenated carbons C-3 as usual. The appearance of the carboxyl signal (-COO-) at $\delta \quad 183.5$ was determined as C-28. Finally, the structure of (1) was established as oleanolic acid by comparison with data in the literature [9]. Oleanolic acid was known to have numerous of pharmacological activities including inhibitory activity against HIV-1 protease with $\mathrm{IC}_{50}$ of 6.3 $\mu \mathrm{M}$ [10], anti-inflammatory, anti-cancer, and hepato-protective effects, that were tested for their ability to modulate the activities of several cytochrome P450 (CYP) enzymes using human liver microsomes [4], inhibition the growth of ras oncogene-transformed R6 cells without toxicity to the normal cells [8]. Additionally, (1) had high yield, about $0.15 \%$ of dried leaves, and this material is easily collected in mangrove forest. So Sonneratia alba should be exploited to afford a valuable source for food and pharmaceutical products.

Compound (2) was a colourless amorphous powder. The ${ }^{13} \mathrm{C}$-NMR spectral data of (2) showed that it was also a triterpene with 30 signals like (1). However, the DEPT spectrum showed that (2) had two carbon signals of a disubstituted double bond at $\delta 109.7\left(=\mathrm{CH}_{2}\right)$ and $150.5(=\mathrm{C}<)$, these data supported (2) to be a lupane type triterpene. Beside an oxygenated methine group at 79.0 of C-3, (2) had another oxygenated methylene carbon signal at 60.6 ppm (C-28), matching with two proton signals $[(3.80, d, 11.0 \mathrm{~Hz}, \mathrm{H}-28 \mathrm{a})$ and $(3.35, d, 11.0$ $\mathrm{Hz}, \mathrm{H}-28 \mathrm{~b})]$. Comparison spectroscopic data of (2) with those in literature [9] suggested that (2) was betulin (lup-20(29)-en-3 $\beta, 28$-diol).

Table 1. NMR data of compounds (1), (2), (3), (4) and related references in CDCl3.

\begin{tabular}{|c|c|c|c|c|c|c|c|c|c|c|c|c|}
\hline \multirow{2}{*}{$\mathbf{N}^{\mathbf{0}}$} & \multicolumn{2}{|l|}{ (1) } & $\left(1^{*}\right)$ & \multicolumn{2}{|l|}{ (2) } & $\left(2^{*}\right)$ & \multicolumn{2}{|l|}{$(3)^{\mathrm{a}}$} & $\left(3^{*}\right)$ & \multicolumn{2}{|l|}{$(4)^{\mathrm{a}}$} & $\left(4^{* a}\right)$ \\
\hline & $\delta_{\mathrm{H}}(\mathrm{J}=\mathrm{Hz})$ & $\delta_{\mathrm{C}}$ & $\delta_{\mathrm{C}}$ & $\delta_{\mathrm{H}}(\mathrm{J}=\mathrm{Hz})$ & $\delta_{\mathrm{C}}$ & $\delta_{\mathrm{C}}$ & $\delta_{\mathrm{H}}(\mathrm{J}=\mathrm{Hz})$ & $\delta_{\mathrm{C}}$ & $\delta_{\mathrm{C}}$ & $\delta_{\mathrm{H}}(\mathrm{J}=\mathrm{Hz})$ & $\delta_{\mathrm{C}}$ & $\delta_{\mathrm{C}}$ \\
\hline 1 & & 38.4 & 38.5 & & 38.7 & 38.8 & & 39.3 & 38.7 & - & 48.2 & 48.2 \\
\hline 2 & & 27.2 & 27.4 & & 27.4 & 27.2 & & 28.3 & 27.4 & $4.08(1 \mathrm{H}, t d, 10.0,4.5)$ & 68.9 & 68.9 \\
\hline 3 & $3.23(1 \mathrm{H}, d, 7.0)$ & 79.0 & 78.7 & $3.18(1 \mathrm{H}, d d, 11.0,5.0)$ & 79.0 & 78.9 & $3.45(1 \mathrm{H}, m)$ & 78.1 & 78.9 & $3.39(1 \mathrm{H}, d, 10.0)$ & 83.8 & 83.8 \\
\hline 4 & & 38.7 & 38.7 & & 38.9 & 38.9 & & 39.5 & 38.8 & & 38.8 & 38.8 \\
\hline 5 & & 55.2 & 55.2 & $0.69(1 \mathrm{H}, d, 10.0)$ & 55.3 & 55.3 & & 55.9 & 55.3 & & 56.1 & 56.1 \\
\hline 6 & & 18.3 & 18.3 & & 18.3 & 18.3 & & 18.8 & 18.3 & & 18.8 & 18.8 \\
\hline 7 & & 32.6 & 32.6 & & 34.3 & 34.3 & & 34.8 & 34.3 & & 34.8 & 34.8 \\
\hline 8 & & 39.3 & 39.3 & & 40.9 & 40.9 & & 41.1 & 40.7 & & 41.2 & 41.2 \\
\hline 9 & & 47.6 & 47.6 & & 50.4 & 50.4 & & 50.9 & 50.5 & & 51.0 & 51.0 \\
\hline 10 & & 37.1 & 37.0 & & 37.2 & 37.2 & & 37.5 & 37.2 & & 39.9 & 39.9 \\
\hline 11 & & 22.9 & 23.1 & & 20.9 & 20.9 & & 21.2 & 20.8 & & 21.4 & 21.4 \\
\hline
\end{tabular}




\begin{tabular}{|c|c|c|c|c|c|c|c|c|c|c|c|c|}
\hline 12 & & 122.6 & 122.1 & & 25.2 & 25.3 & & 26.1 & 25.5 & & 26.1 & 26.1 \\
\hline 13 & & 143.6 & 143.4 & & 37.4 & 37.3 & & 38.6 & 38.4 & & 38.6 & 38.6 \\
\hline 14 & & 41.6 & 41.6 & & 42.7 & 42.7 & & 42.8 & 42.4 & & 42.9 & 42.9 \\
\hline 15 & & 27.7 & 27.7 & & 27.1 & 27.0 & & 31.2 & 30.5 & & 30.2 & 30.2 \\
\hline 16 & & 23.4 & 23.4 & & 29.2 & 29.2 & & 32.9 & 32.1 & & 32.9 & 32.9 \\
\hline 17 & & 46.5 & 46.6 & & 47.8 & 47.8 & & 56.6 & 56.3 & & 56.6 & 56.7 \\
\hline 18 & $2.83(1 \mathrm{H}, d, 10.0)$ & 41.0 & 41.3 & & 48.8 & 48.8 & $3.53(1 \mathrm{H}, m)$ & 47.7 & 46.8 & & 49.8 & 49.8 \\
\hline 19 & & 45.8 & 45.8 & & 47.8 & 47.8 & & 49.8 & 49.2 & & 47.8 & 47.8 \\
\hline 20 & & 30.7 & 30.6 & & 150.5 & 150.6 & & 151.3 & 150.3 & & 151.3 & 151.3 \\
\hline 21 & & 33.8 & 33.8 & & 29.8 & 29.8 & & 30.3 & 29.7 & & 31.2 & 31.2 \\
\hline 22 & & 32.4 & 32.3 & & 34.0 & 34.0 & & 37.6 & 37.0 & & 37.6 & 37.6 \\
\hline 23 & $0.90(3 \mathrm{H}, s)$ & 28.1 & 28.1 & $0.97(3 \mathrm{H}, s)$ & 28.0 & 28.0 & $1.22(3 \mathrm{H}, s)$ & 28.6 & 27.9 & $1.05(3 \mathrm{H}, s)$ & 29.2 & 29.2 \\
\hline 24 & $0.93(3 \mathrm{H}, s)$ & 15.5 & 15.6 & $0.76(3 \mathrm{H}, s)$ & 15.4 & 15.4 & $0.83(3 \mathrm{H}, s)$ & 16.3 & 15.3 & $0.90(3 \mathrm{H}, s)$ & 17.7 & 17.7 \\
\hline 25 & $1.13(3 \mathrm{H}, s)$ & 15.3 & 15.3 & $1.02(3 \mathrm{H}, s)$ & 16.1 & 16.1 & $1.07(3 \mathrm{H}, s)$ & 16.4 & 16.0 & $1.04(3 \mathrm{H}, s)$ & 17.4 & 17.4 \\
\hline 26 & $0.92(3 \mathrm{H}, s)$ & 17.1 & 16.8 & $0.83(3 \mathrm{H}, s)$ & 16.0 & 16.0 & $1.00(3 \mathrm{H}, s)$ & 16.4 & 16.1 & $1.04(3 \mathrm{H}, s)$ & 16.5 & 16.5 \\
\hline 27 & $0.99(3 \mathrm{H}, s)$ & 26.0 & 26.0 & $0.98(3 \mathrm{H}, s)$ & 14.8 & 14.8 & $1.06(3 \mathrm{H}, s)$ & 14.9 & 14.7 & $1.25(3 \mathrm{H}, s)$ & 14.9 & 14.9 \\
\hline 28 & & 183.5 & 181.0 & $\begin{array}{c}3.79(1 \mathrm{H}, d d, 11.0 \\
2.0) \\
3.34(1 \mathrm{H}, d, 11.0)\end{array}$ & 60.6 & 60.2 & - & 178.9 & 180.5 & & 178.9 & 179.1 \\
\hline 29 & $0.78(3 \mathrm{H}, s)$ & 33.1 & 33.1 & $\begin{array}{l}4.68(1 \mathrm{H}, \text { brs }) \\
4.58(1 \mathrm{H}, \text { brs })\end{array}$ & 109.6 & 109.6 & $\begin{array}{c}4.94(1 \mathrm{H}, d, \\
2.5) \\
4.76(1 \mathrm{H}, b r s)\end{array}$ & 109.9 & 109.6 & $\begin{array}{l}4.92(1 \mathrm{H}, d, 2.0) \\
4.76(1 \mathrm{H}, d, 1.5)\end{array}$ & 109.4 & 109.9 \\
\hline 30 & $0.76(3 \mathrm{H}, s)$ & 23.6 & 23.6 & $1.68(3 \mathrm{H}, s)$ & 19.1 & 19.1 & $1.79(3 \mathrm{H}, s)$ & 19.5 & 19.4 & $1.77(3 \mathrm{H}, s)$ & 19.5 & 19.5 \\
\hline
\end{tabular}

Compound (3) was a colourless amorphous powder. The ${ }^{13} \mathrm{C}$ and DEPT-NMR spectral data of (3) and (2) are similar with 30 signals of a triterpene including two olefinic carbon signals at $\delta 109.9\left(=\mathrm{CH}_{2}\right)$ and $151.3(=\mathrm{C}<)$, one oxygenated methine carbon signal at $\delta 78.1$ as usual. It matched with two olefinic proton signals at $\delta 4.94$ and 4.76 and six singlet methyl proton signals at $\delta 1.8$ to 0.8 . However, (3) had a carboxyl signal at $\delta 178.9$ (C-28) instead of an oxygenated methylene carbon signal. So (3) was a triterpenoid acid with a lupane skeleton. Moreover, the NMR spectral data of (3) showed a good compatibility to those in reference [9], so (3) was proposed to be betulinic acid (3 $\beta$-hydroxylup-20(29)-en-28oic acid). (3) exhibited inhibitory activities against HIV-1 replication in acutely infected H9 lymphocyte cells with $\mathrm{EC}_{50}$ values of 1.4 $\mu \mathrm{M}$ and $\mathrm{TI}$ values of $9.3 \mu \mathrm{M}$ [2]. This compound was selectively cytotoxic against several human melanoma cancer cell lines $\left(\mathrm{MEL}-1 \quad \mathrm{ED}_{50}=1.1 \mu \mathrm{g} / \mathrm{ml}, \mathrm{MEL}-2 \mathrm{ED}_{50}=2.0\right.$ $\mu \mathrm{g} / \mathrm{ml}$, and MEL-4 ED $\left.{ }_{50}=4.8 \mu \mathrm{g} / \mathrm{ml}\right)$. Betulinic acid was then found to be active in vivo test using athymic mice carrying human melanomas, with a slight toxicity. Further biological studies indicated that betulinic acid works by induction of apoptosis [7].

Compound (4) was a colourless amorphous powder. The ${ }^{13} \mathrm{C}$ and DEPT-NMR spectral data of (4) and (3) were similar with 30 signals of a lupane skeleton including two olefinic carbon signals at $\delta 109.9\left(=\mathrm{CH}_{2}\right)$ and $151.3(=\mathrm{C}<)$, one

\section{Trang 14}


oxygenated methine carbon signal at $\delta 83.81$ (C-3) as usual, one carboxyl signal at $\delta 178.9$ (C-28). It matched with two olefinic proton signals at $\delta 4.92$ and 4.76 and six singlet methyl proton signals at $\delta 1.8$ to 0.8 . However, (4) had one more oxygenated methine carbon signal at $\delta 68.9(\mathrm{C}-2)$ in ${ }^{13} \mathrm{C}$ spectrum, as well as one oxygenated proton signal at $\delta 4.08(1 \mathrm{H}$, $\mathrm{td}, 10.0,4.5, \mathrm{H}-2)$ in ${ }^{1} \mathrm{H}$ spectrum. The large coupling constant of $\mathrm{H}-2$ supported that the hydroxyl group at C-2 had $\alpha$-orientation. So (4) was alphitolic acid ( $2 \alpha, 3 \beta$-dihydroxylup20(29)-en-28-oic acid) via comparison with data in literature [4].

Compound (5) was a colourless amorphous powder. The ${ }^{13} \mathrm{C}$ and DEPT spectra showed that (5) had some carbon signals of one aromatic ring including two oxygenated carbon signals at $\delta 146.5$ (C-3, C-5), 139.8 (C-4), a methine carbon signal at $\delta 110.1$ (C-2, C-6), a quaternary carbon signal at $\delta 121.6$ (C1). Besides that, it had a carboxyl carbon signal at $\delta 169.1$ and an oxygenated methyl carbon signal at $\delta 52.3$. It corresponded to an aromatic proton signal at $\delta 7.01(2 \mathrm{H}, \mathrm{s})$ and an oxygenated methyl proton signal at $\delta 3.82(3 \mathrm{H}$, $s$ ) in ${ }^{1} \mathrm{H}-\mathrm{NMR}$ spectrum. So (5) was methyl gallate through the comparison with data in literature [3].

Compound (6) was a colourless amorphous powder. Its ${ }^{1} \mathrm{H}-\mathrm{NMR}$ spectrum showed a proton signal at $\delta 9.60(1 \mathrm{H}, s)$ of a formyl group, an oxygenated proton signal at $\delta 4.91$ $(2 \mathrm{H}, s)$, two doublet aromatic proton signals with small coupling contanst of $3.0 \mathrm{~Hz}$ at $\delta 7.39$ and 6.59 of an aromatic ring with two adjacent-hydrogens. Furthermore, ${ }^{13} \mathrm{C}$ and DEPT spectra of (6) showed one signal at $\delta 178.2$ of $-\underline{\mathbf{C H}}=\mathrm{O}$, one oxygenated methylene carbon signal at $57.5 \mathrm{ppm}$ and four aromatic carbon signals of a furane ring. Comparison the spectral data of compound (6) with those in the literature [6] showed a good compatibility, so the structure of (6) was elucidated as 5hydroxymethylfurfural.

\section{CONCLUSION}

From the fresh leaves of Sonneratia alba J.E. Smith collected in Viet Nam; oleanolic acid (1), betulin (2), betulinic acid (3), alphitolic acid (4), methyl gallate (5) and 5hydroxymethylfurfural (6) were isolated sucessfully. Further studies on this plant are in progress. 


\title{
THÀNH PHẦ HÓA HỌC CỦA LÁ CÂY BẦ TRÁNG SONNERATIA ALBA J.E. SMITH., HỌ BẦ (SONNERATIACEAE)
}

\author{
Nguyễn Thị Hoài Thu ${ }^{(1)}$, Lâm Phục Khánh ${ }^{(1)}$, Nguyễn Thế Duy ${ }^{(1)}$, Nguyễn Thị Kim Chánh ${ }^{(1)}$, \\ Nguyễn Kim Phi Phụng ${ }^{(1)}$, Poul Erik Hansen ${ }^{(2)}$ \\ (1) Ttrường Đại học Khoa Học Tự Nhiên, ĐHQG-HCM
}

(2) Đại học Roskilde, Đan Mạch

TÓM TÁT: Cây Bần trắng là loài cây đặc hữu của rùng ngập mặn. Mặc dù đã có khá nhiều nghiên cưu trên các cây ngập mặn, tuy nhiên cây Bần trắng chua được nghiên cứu nhiều trên thế giới. Ỏ Việt Nam, loài này chưa được tác giả nào khảo sát, nên cây Bần trắng được chọn làm đối tuợng nghiên cứu của đề tài này. Tù̀ cao eter dầu hỏa của lá cây Bần trắng, 6 hợp chất đã được cô lập gồm acid oleanolic (1), betulin (2), acid betulinic (3), acid alphitolic (4), methyl gallat (5) và 5hydroxymethylfurfural (6). Cấu trúc hóa học của các hợp chất này được xác định dụa trên các phuơng pháp phổ nghiệm kết hợp so sánh với số liệu trong tài liệu tham khảo. Trong số bốn hợp chất trên, acid oleanolic hiện diện với hàm luợng cao, khoảng 0.15\% so với bột lá khô. Các kết quả nghiên cứu cho thấy acid oleanolic có hoạt tính mạnh kháng HIV và kháng ung thu. Điều này định huoóng cho việc khai thác lá Bần (nếu không thu hái lá cũng rụng bỏ), thu lấy acid oleanolic, chế biến thành các loại thực phẩm và duợc phẩm có giá trị. Các nghiên cứu tiếp theo trên cây này vẫn đang được tiếp tục.

Tù khóa: Sonneratiaceae, Sonneratia alba, acid oleanolic, betulin, acid betulinic, acid alphitolic, methyl gallat, 5-hydroxymethylfurfural.

\section{REFERENCES}

[1]. Vo Van Chi, Dictionary of Vietnamese medicinal plants, Medicine Publishing House, 78, 724 (1997).

[2]. Fumio Hashimoto, Yoshiki Kashiwada, L. Mark Cosentino, Chin-Ho Chen, Patricia E. Garrett and Kuo-Hsiung Lee, Anti-AIDS agents-XXVII: synthesis and anti-HIV activity of betulinic acid and dihydrobetulinic acid derivatives, Bioorganic \& Medicinal Chemistry, 5, 2133-2143 (1997).
[3]. Ji Young Oh, Ung Choi, Yong Suk Kim, Dong Hwa Shin, Isolation and indentification of antioxidative components from bark of Rhus javanica Linne, . Korean J. Food Sci. Technol., 35, 726732 (2003).

[4]. Jia Hao, Xueli Zhang, Pu Zhang, Jun Liu, Luyong Zhang, Hongbin Sun, Efficient access to isomeric 2,3dihydroxy lupanes: first synthesis of alphitolic acid, Tetrahedron, 65, 79757984 (2009).

[5]. Kyoung-Ah Kim, Ji-Suk Lee, Hi-Joon Park, Jin-Woo Kim, Chang-Ju Kim, In- 
Sop Shim, Nam-Jae Kim, Seung-Moo

Han, Sabina Lim, Inhibition of cytochrome P450 activities by oleanolic acid and ursolic acid in human liver microsomes, Life Sciences 74, 27692779 (2004).

[6]. Mitisuo Miyazawa, Jun Anzai, Jun Fujioka and Yukio Isikawa, Insecticidal Compounds against Drosophila Melanogaster from Cornus Officinalis Sieb. Et Zucc, Natural Product Research, 17, 337-339 (2003).

[7]. Pish, E., Chai H., Lee I.S., Chagwedera T.E., Farnsworth N.R., Cordell G.A., Beecher C.W., Fong H.H., Kinghorn A.D., Brown D.M., Wani M.C., Wall M.E., Hieken T.J., Das Gupta T.K., Pezzuto J.M., Discovery of betulinic acid as a selective inhibitor of human melanoma that functions by induction of apoptosis, Nature Medicine 1, 10461051, (1995).

[8]. Pui Kei Wu, William Chi Shing Tai, Zhi-Tao Liang, Zhong-Zhen Zhao, Wen Luan Wendy Hsiao, Oleanolic acid isolated from Oldenlandia diffusa exhibits a unique growth inhibitory effect against ras-transformed fibroblasts, Life Sciences 85, 113-121 (2009).

[9]. Shasshi B. Mahato, Asish P. Kyndu, ${ }^{13}$ C-NMR spectra of pentacyclic triterpeneoids - A compilation and some salient features, Phytochemistry, 37, 1517-1575 (1994).

[10]. Ying Wei, Chao-Mei Ma, Masao Hattori, Anti-HIV protease triterpeneoids from the acid hydrolysate of Panax ginseng, Phytochemistry Letters 2, 63-66 (2009). 